\title{
Gaucher disease in Romanian patients: incidence of the most common mutations and phenotypic manifestations
}

\author{
Cristina Drugan*, ${ }^{*}$, Lucia Procopciuc ${ }^{1}$, Gheorghe Jebeleanu ${ }^{1}$, Paula Grigorescu-Sido ${ }^{2}$, \\ Jane Dussau ${ }^{3}$, Livia Poenaru ${ }^{3}$ and Catherine Caillaud ${ }^{3}$
}

\author{
${ }^{1}$ Department of Biochemistry, University of Medicine and Pharmacy Cluj-Napoca, Romania; ${ }^{2}$ Department of \\ Paediatrics, University of Medicine and Pharmacy Cluj-Napoca, Romania; ${ }^{3}$ Department of Genetics, Paris $V$ \\ University, Institut Cochim, Paris, France
}

Gaucher disease (GD) is an inherited glycolipid storage disorder resulting from the deficiency of glucocerebrosidase. It is the most frequent lysosomal storage disease in Romania, accounting for $70 \%$ of all lysosomal disorders diagnosed since 1997 in this country. The prevalence of six common mutations (N370S, L444P, R463C, 84GG, recNcil and recTL) and their phenotypic impact were studied in 20 type 1 GD patients of non-Jewish origin. Mutation analysis identified $77.8 \%$ of the GD alleles. The N370S mutation had the highest prevalence (50\%), followed by the L444P $(22.2 \%)$ and the recNcil $(5.6 \%)$ alleles. Mutations R463C, 84GG and recTL have not been found in our patients. Rare or novel mutations likely accounted for $\mathbf{2 2 . 2 \%}$ of the disease-producing uncharacterised alleles. Our study indicates a high prevalence of type 1 among Romanian GD patients. Clinical phenotype and disease severity were evaluated according to the standardised severity score index. Genotype-phenotype correlations were similar to those reported for other Caucasian non-Jewish populations. The absence of neuronopathic disease in patients presenting at least one copy of the N370S allele was confirmed, but the relative mildness of N370S homozygotes was not a constant feature among our patients. The presence of the L444P or of uncharacterised sporadic mutations was always associated with severe clinical manifestations, even in compound heterozygotes with the N370S allele. A large degree of phenotypic variability was observed in patients displaying the same genotype. The particularities of genotype-phenotype correlations may suggest the impact of other genetic or non-genetic factors on the clinical picture. European Journal of Human Genetics (2002) 10, 511 - 515. doi:10.1038/sj.ejhg.5200845

Keywords: Gaucher disease; mutation analysis; genotype-phenotype correlations

\section{Introduction}

Gaucher disease (GD) is an autosomal recessive sphingolipidosis caused by a deficiency of the lysosomal hydrolase acid $\beta$-glucosidase (glucocerebrosidase, E.C. 3.2.1.45). It results in a progressive accumulation of glucosylceramide in the lysosomes of macrophages, leading to hepatosplenomegaly, anemia, thrombocytopenia and various skeletal complica-

\footnotetext{
*Correspondence: C Drugan; Department of Biochemistry, University of Medicine and Pharmacy, Str Splaiul Independenei 4/7, 3400 Cluj-Napoca Romania. Tel: +40-95-617634; Fax: +40-64-193847;

E-mail: cdrugan@umfcluj.ro

Received 27 December 2001; revised 1 May 2002; accepted 13 May 2002
}

tions. GD has a striking phenotypic variability, with respect to age of onset, progression and severity. However, three main clinical types have been delineated according to the absence (type 1 , non neuronopathic) or the presence (type 2 , acute neuronopathic and type 3 , subacute neuronopathic) of neurological involvement. ${ }^{1}$ To date, more than 120 disease-producing alleles have been described, but only a few point mutations (N370S, 84GG, L444P, IVS2+1g $>$ a, $\mathrm{D} 409 \mathrm{H}$ ) and complex alleles (resulting from genetic rearrangements between the functional gene and the closely located pseudogene) occur with significant frequencies in 
GD patients. ${ }^{2}$ The prevalence of different mutations has been extensively studied and some useful genotype-phenotype correlations have been inferred., ${ }^{3,4}$ Although the predictive value of these correlations is generally limited, their study in different ethnic groups had a significant impact on genetic counselling and on the better understanding of the molecular aspects of GD.

We report here the first study of mutations causing GD in Romanian patients. The biochemical diagnosis of GD was introduced in 1997 in this country, based on the measurement of glucocerebrosidase activity in peripheral blood leukocytes. Since then, GD has been the most frequent (70\%) lysosomal storage disorder diagnosed in our laboratory. Twenty patients belonging to 18 different families were tested for the presence of six known mutations (N370S, L444P, R463C, 84GG, recNciI and recTL). The aim of this study was to establish the most frequent mutations in our group of patients and to analyse their phenotypic impact.

\section{Materials and Methods \\ Patients}

Patients originated from various regions of Romania. All of them declared a non-Jewish ethnic origin. Clinical evaluation included haematological tests, investigation of liver and spleen function, investigation of the presence and the extent of organomegaly, ophthalmologic examination and evaluation of skeletal and neurological involvement (clinical and electro-encephalographic examination). The degree of clinical severity was established according to the severity score index (SSI) proposed by Zimran et al. ${ }^{5}$ As enzyme replacement therapy is just starting in Romania, the clinical status of the patients reflected the natural course of GD, prior to any specific treatment. Measurement of glucocerebrosidase activity in leukocytes was performed using the artificial substrate 4-methylumbelliferyl- $\beta$-Dglucopyranoside. ${ }^{6}$

\section{Detection of mutations}

High molecular weight genomic DNA was extracted from peripheral blood leukocytes according to standard methods.

Mutation N370S was detected by mismatched PCR amplification, as previously described, ${ }^{7}$ with minor modifications. Primer sequences were extended (5'-GTCTCTTTGCCTTTGTCCTTACCCTCGGA-3' for the upstream primer and 5'-ACTGTCGACAAAGTTACGCACCCAAT-3' for the downstream primer) yielding a more specific amplification of a $120 \mathrm{bp}$ fragment bearing the N370S mutation. The base changed to create an XhoI site is underlined. Mutation 84GG was also studied by mismatched PCR amplification, as previously described. ${ }^{8}$

Mutations L444P and R463C were studied by selective amplification of the corresponding DNA fragment, followed by MspI digestion, according to the method previously described. ${ }^{9}$ The recombinant alleles recNcil (including mutations L444P, A456P and V460V) and recTL (including mutations D409H, L444P, A456P and V460V) were analysed by sequencing of the amplified fragments bearing the L444P substitution. PCR products were purified using the QIAquick PCR purification kit (Qiagen). The sequencing reactions were performed on an automated ABI 373A DNA sequencer with the Big Dye Terminator Ampli Taq FS kit (ABI), according to the manufacturer's recommendations.

Biochemical and molecular testing of the family members (siblings and both parents) were conducted for each index case, in order to identify possible asymptomatic relatives and to ensure the correct genotyping of homozygotes.

\section{Results}

\section{Patient characteristics}

The studied group consisted of 20 non-Jewish Gaucher disease patients (14 females and six males, with ages ranging from 2 to 53 years), displaying a marked reduction of $\beta$-glucocerebrosidase activity. Their most important clinical features are summarised in Table 1. Forty-five per cent (nine out of 20) previously underwent splenectomy, whereas eight of the remaining patients (40\%) had splenomegaly. Hepatomegaly was observed in 17 patients $(85 \%)$. Thrombocytopenia was present in 10 patients (50\%) and anemia in 16 patients (80\%). Bone abnormalities of different grades have been found in $95 \%$ of the patients and included Erlenmeyer flask deformities (90\%), chronic bone pain $(65 \%)$, osteoporosis/osteolysis $(30 \%)$, pathologic fractures/hip replacement (20\%). All the patients were classified as having the non-neuronopathic (type 1) form of the disease, according to the absence of any signs of neurological involvement.

\section{Mutation analysis}

Screening for the most frequent mutations allowed the identification of $77.8 \%$ of the mutant alleles. Among the six common mutations tested, only three were found in this study: N370S (50\%), L444P (22.2\%) and recNciI $(5.6 \%)$. Sporadic uncharacterised mutations accounted for $22.2 \%$ of the mutant alleles. Mutations R463C, 84GG and recTL were not found in our patients. Since our group of patients included two pairs of siblings, the frequencies were calculated on a total number of 36 alleles.

Genotypes of the patients are presented in Table 1. Most of them comprised the N370S mutation, either in a homozygous state (three patients), or in association with the L444P or recNciI alleles (eight patients), or with an uncharacterised mutation (seven patients). Only one patient was homozygous for the L444P mutation and one was a compound heterozygote for the L444P and an unknown mutation.

\section{Genotype-phenotype correlations}

The majority of the patients, whatever their genotype, exhibited various degrees of haematological and skeletal 
Table 1 Genotypes and clinical features of Romanian Gaucher patients

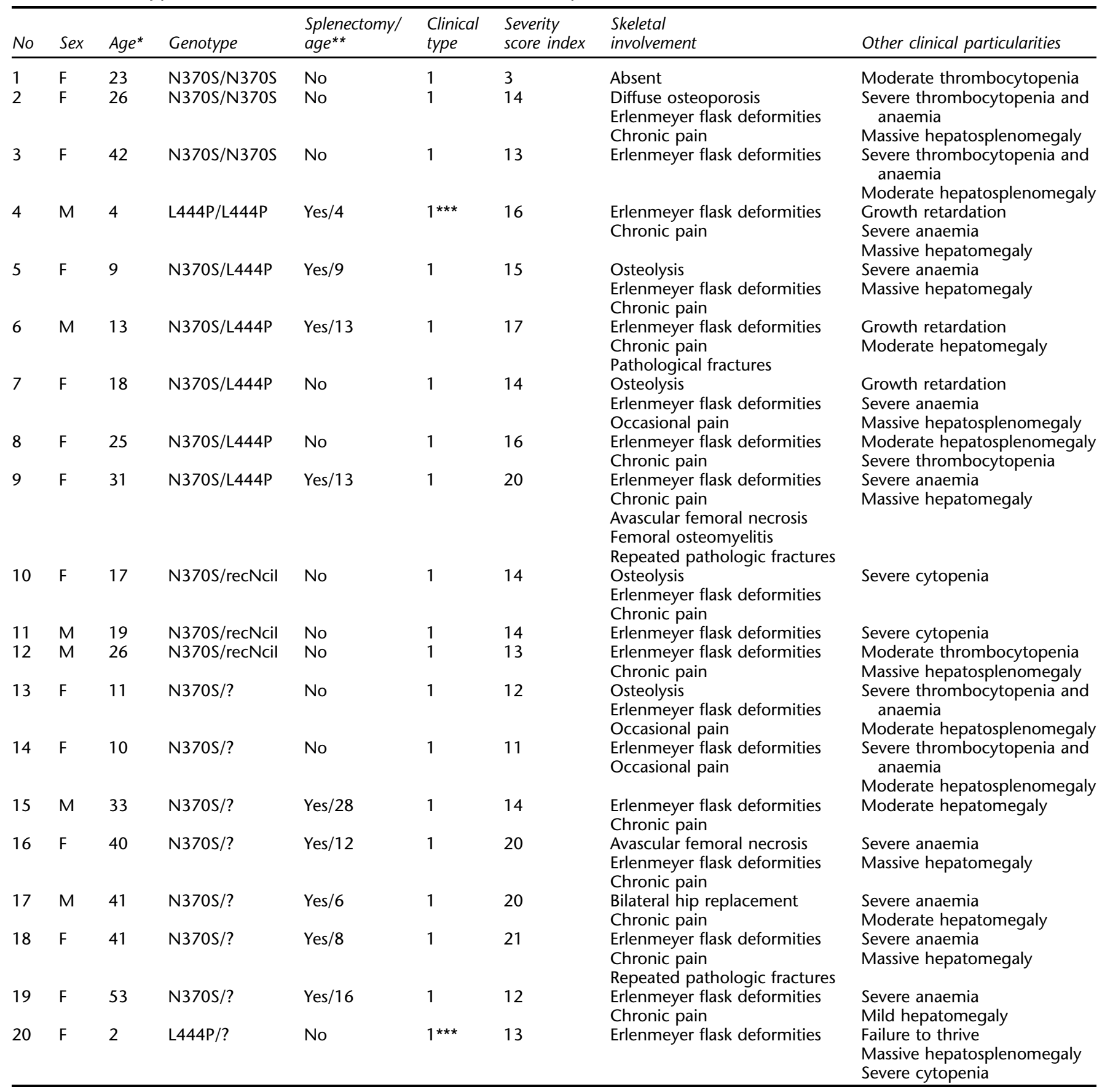

${ }^{*}$ The age (expressed in years) corresponds to the moment of enzymatic diagnosis and has not been strictly considered as a criterion of disease severity, because all patients were formally diagnosed after 1997, when the enzymatic assay became available. Therefore, this parameter cannot reflect the real age at which the diagnosis would have been established. ${ }^{* *}$ The age at splenectomy may be anterior to the age at which the enzymatic diagnosis was established. ${ }^{* * *}$ No signs of neurological involvement were detected in patients no 4 and 20 at the age of 8 and 4 years old, respectively. Patients no $1-2$ and 10-11 are siblings. The severity score index was calculated according to Zimran et al ${ }^{5}$.

involvement, as reflected by the SSI values ranging between 11 and 21 . The most severe bone complications (multiple fractures, hip replacement) were found in two patients carrying the N370S mutation, in asso- ciation with the L444P or with an uncharacterised mutation.

One patient homozygous for the N370S mutation (no. 1) was nearly asymptomatic (SSI=3), even though her sister 
(no. 2) exhibited hepatosplenomegaly, pancytopenia and bone involvement (SSI=14). The two patients carrying the L444P mutation, either in a homozygous state, or in association with an unknown mutation, had no neurological involvement, either at the time of diagnosis, or at a subsequent re-evaluation at the age of 8 and 4 , respectively.

\section{Discussion}

The distribution of mutant alleles identified in our patients was compared with data originating from different populations. The frequency of the N370S allele in Romanian GD patients $(50 \%)$ was higher than that reported in other non-Jewish European populations, ${ }^{10,11}$ except Portuguese patients. $^{12}$ The second most prevalent mutation, L444P, was responsible for $22.2 \%$ of the mutant alleles, a frequency comparable to that reported for other non-Jewish populations $(25 \%){ }^{2}$ The absence of the $84 \mathrm{GG}$ mutation is not surprising, as this mutation is considered to be specific for Jewish GD patients. ${ }^{13}$ The distribution of common mutations in Romanian GD patients was close to that established for Czech and Slovak patients, ${ }^{14}$ but different from another East European country, Poland, ${ }^{15}$ where the L444P substitution is the most prevalent mutation, due to a relatively high frequency of type 3 disease. Our results show some similarities with other non-Jewish EastEuropean populations, ${ }^{14,15}$ but also indicate a high prevalence of common mutations (77.8\%), which may be related to the higher ethnic homogeneity of our population.

The presence of two siblings homozygous for the N370S allele with markedly different clinical presentation supports the observed intra-familial heterogeneity of GD patients, indicating that other genetic or non-genetic factors may contribute to the clinical picture of the disease. ${ }^{16}$ The asymptomatic sibling was diagnosed by family investigations following the identification of the index case, suggesting that other asymptomatic N370S homozygotes may exist in our population, but do not come to medical attention, as previously shown by Zimran et $a l^{17}$ in the Ashkenazi Jewish population.

In our study, the presence of the N370S mutation was always associated with non-neuronopathic disease. These results are similar to those previously reported, concerning the protective role of at least one N370S allele with respect to the development of neurological signs. ${ }^{18}$ However, patients who were compound heterozygotes for the N370S allele and an unknown mutation displayed a large degree of clinical variability (SSI values ranging between 11 and 21). This group included the patients who were most severely affected by the skeletal and haematological complications of GD. These data are different from those previously described in other non-Jewish populations, indi- cating a correlation between at least one N370S allele and a milder course of the disease. ${ }^{19}$

The L444P mutation may be associated with all types of Gaucher disease. In our study, most of the patients carrying the L444P mutation were compound heterozygotes for this allele and the N370S mutation. A constant feature was the presence of severe skeletal involvement (SSI values between 13 and 20) and a more severe course of the disease, including delayed growth in children.

No neuronopathic involvement has been demonstrated in the patient homozygous for the L444P allele, either at the time of diagnosis or at a subsequent follow-up examination at the age of 8, which included an electroencephalographic and ophthalmologic evaluation. However, as an electro-oculographic recording of eye movements was not available, the presence of subclinical oculomotor abnormalities or the future evolution towards the subacute neuronopathic type 3 GD cannot be excluded in this patient, although homozygosity for the L444P mutation has already been found associated to type $1 \mathrm{GD}^{20}$

One patient was heterozygous for the L444P allele and an unknown mutation. This patient died at the age of 4 , following complications of massive hepatosplenomegaly, but again no neurological involvement could be demonstrated. The clinical picture may suggest a possible type $3 \mathrm{~b}$ variant, in which death may have occurred before the involvement of the central nervous system became detectable. $^{21}$

Our study provides an insight into the molecular bases of GD in Romanian patients. The distribution of mutant alleles and the frequency with which particular genotypes were encountered displayed some specific particularities, which may be related to the ethnic characteristics of our population. Genotype-phenotype correlations confirmed the previously reported 'protective' role of the N370S allele against the development of neurological complications and also indicated a more severe phenotypic impact of the L444P allele and of some sporadic, uncharacterised, mutations. Phenotypic variation was described among patients with identical genotypes, confirming that other genetic, environmental or developmental factors may contribute to the clinical expression of GD. Understanding the natural history of the disease and the clinical consequences of the different genotypes in our patients is critical to the establishment of procedures for monitoring and treating this lifelong disorder.

\section{Acknowledgement}

This study was supported by grants from Genzyme GmbH and the Romanian Ministry of Education. 


\section{References}

1 Beutler E, Grabowski GA: Gaucher disease; in Scriver CR, Beaudet AL, Sly WS, Valle D (eds) The Metabolic and Molecular Bases of Inherited Disease. 7th edn. New York: McGraw-Hill, 1995; pp $2641-2670$.

2 Grabowski GA, Horowitz M: Gaucher's disease: molecular, genetic and enzymological aspects; in Zimran A (ed) Baillière's Clinical Haematology International Practice and Research: Gaucher's Disease. Baillière Tindall, 1997; vol 10/no 4, pp 635-656.

3 Sidransky E, Bottler A, Stubblefield B, Ginns EI: DNA mutational analysis of type 1 and type 3 Gaucher patients: how well do mutations predict phenotype? Hum Mutat 1994; 3: 25-28.

4 Koprivika V, Stone DL, Park JK et al: Analysis and classification of 304 mutant alleles in patients with type 1 and type 3 Gaucher disease. Am. J Hum Genet 2000; 66: 1777-1786.

5 Zimran A, Kay A, Gelbart T et al: Gaucher disease. Clinical, laboratory, radiologic and genetic features of 53 patients. Medicine 1992; 71: $337-353$.

6 Peters SP, Coyle P, Glew RH: Differenciation of beta-glucocerebrosidase from beta-glucosidase in human tissues using sodium taurocholate. Arch Biochem Biophys 1976; 175: 569-582.

7 Beutler E, Gelbart T, West C: The facile detection of the nt 1226 mutation of glucocerebrosidase by mismatched PCR. Clin Chim Acta 1990; 194: $161-166$.

8 Beutler E, Gelbart T, Kuhl W, Sorge J, West C: Identification of the second common Jewish Gaucher disease mutation makes possible population-based screening for the heterozygous state. Proc Natl Acad Sci USA 1991; 88: 10544-10547.

9 Dahl N, Lagerström M, Erikson A, Pettersson U: Simplified detection of Nci mutation in Gaucher disease. Lancet 1990; 335: 1589 1590.

10 Michelakakis H, Dimitriou E, Van Weely S et al: Characterisation of glucocerebrosidase in Greek Gaucher disease patients: mutation analysis and biochemical studies. J Inherit Metab Dis 1995; 18: 609-615.

11 Le Coutre P, Demina A, Beutler E, Beck M, Petrides PE: Molecular analysis of Gaucher disease: distribution of eight mutations and the complete gene deletion in 27 patients from Germany. Hum Genet 1997; 99: 816-821.
12 Amaral O, Lacerda L, Santos R, Pinto RA, Aerts H, Sa Miranda MC: Type 1 Gaucher disease: molecular, biochemical and clinical characterisation of patients from northern Portugal. Biochem Med Metab Biol 1993; 49: 97-107.

13 Horowitz M, Zimran A: Mutations causing Gaucher disease. Hum Mutat 1994; 3: 1-11.

14 Hodanov K, Hrebicek M, Cervenkov M, Mrzov L, Veprekov L, Zemen J: Analysis of the beta-glucocerebrosidase gene in Czech and Slovak Gaucher patients: mutation profile and description of six novel mutant alleles. Blood Cells Mol Dis 1999; 25: 287 298.

15 Tylki-Szymanska A, Millat G, Maire I, Czartoryska B: Types I and III Gaucher disease in Poland: incidence of the most common mutations and phenotypic manifestations. Eur J Hum Genet 1996; 4: 334-337.

16 Amaral O, Fortuna AM, Lacerda L, Pinto RA, Sa Miranda MC: Molecular characterisation of type 1 Gaucher disease families and patients: intra-familial heterogeneity at the clinical level. $J$ Med Genet 1994; 31: 401-404.

17 Zimran A, Gelbart T, Westwood B, Grabowski GA, Beutler E: High frequency of the Gaucher disease mutation at nucleotide 1226 among Ashkenazi Jews. Am J Hum Genet 1991; 49: 855-859.

18 Sibille A, Eng CM, Kim SJ, Pastores G, Grabowski GA: Phenotype/ genotype correlations in Gaucher disease type 1: clinical and therapeutic implications. Am. J Hum Genet 1993; 52: 1094-1101.

19 Walley AJ, Barth ML, Ellis I, Fensom AH, Harris A: Gaucher's disease in the United Kingdom: screening non-Jewish patients for the two common mutations. J Med Genet 1993; 30: 280-283.

20 Masuno M, Tomatsu S, Sukegawa K, Orii T: Non-existence of a tight association between a 444 leucine to proline mutation and phenotypes of Gaucher disease: high frequency of a NciI polymorphism in the non-neuronopathic form. Hum Genet 1990; 84: 203-206.

21 Brady RO, Barton NW, Grabowski GA: The role of neurogenetics in Gaucher disease. Arch Neurol 1993; 50: 1212-1224. 